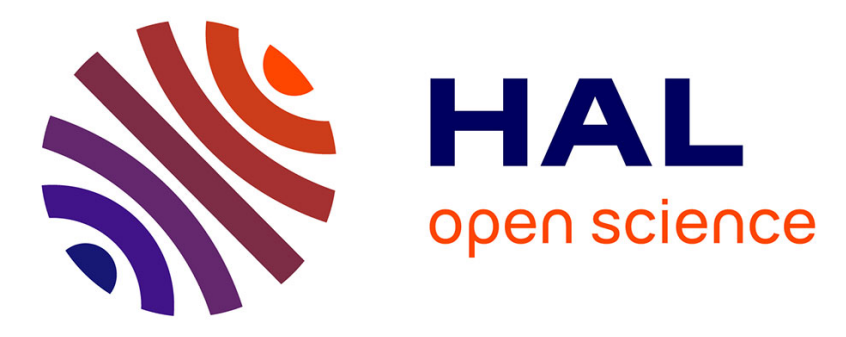

\title{
Fiber Orientation Distribution from Non-Negative Sparse Recovery
}

Thinhinane Megherbi, Aurobrata Ghosh, Fatima Oulebsir Boumghar, Rachid

Deriche

\section{- To cite this version:}

Thinhinane Megherbi, Aurobrata Ghosh, Fatima Oulebsir Boumghar, Rachid Deriche. Fiber Orientation Distribution from Non-Negative Sparse Recovery. MICCAI 2014 Workshop on Computational Diffusion MRI, Sep 2014, Boston, United States. hal-01104163

\section{HAL Id: hal-01104163 \\ https://hal.science/hal-01104163}

Submitted on 19 Jan 2015

HAL is a multi-disciplinary open access archive for the deposit and dissemination of scientific research documents, whether they are published or not. The documents may come from teaching and research institutions in France or abroad, or from public or private research centers.
L'archive ouverte pluridisciplinaire HAL, est destinée au dépôt et à la diffusion de documents scientifiques de niveau recherche, publiés ou non, émanant des établissements d'enseignement et de recherche français ou étrangers, des laboratoires publics ou privés. 


\section{FOD from Non-Negative Sparse Recovery}

- Fiber-orientations modelled as rank-1 tensors: $\mathcal{C}=\lambda c^{\otimes 2 n}$

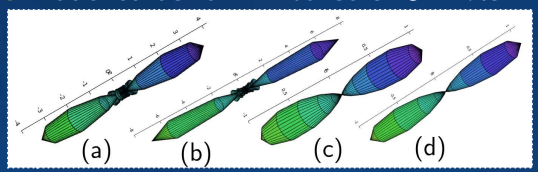

Dirac delta as: (a) SH-8, (b) SH-12, (c) rank1-16, (d) rank1-24

- FOD tensor modelled as the sum of $r$ (unknown) rank-1 tensors:

$$
\mathbf{F}=\sum_{i=1}^{r} \lambda_{i} \mathbf{C}_{i}^{\otimes 2 n}
$$

- Discretize the FOD convolution integral $S(\mathbf{q})=\int_{S^{2}} R(\mathbf{q}, \mathbf{u}) F(\mathbf{u}) d \mathbf{u}$ :

$$
\min _{\{w\}}\|\mathbf{B w}-\mathbf{s}\|^{2} \quad \text { st. } \quad w \geq 0, \quad\left(s_{i}=S_{i}, w_{i}=\lambda_{i}\right)
$$

- Non-Negative Least Squares: find $w$ with sparsity \& non-negativity. (start with $r_{0}=321$ and recover $r$ from sparsity: [Ghosh 2013, 2014])

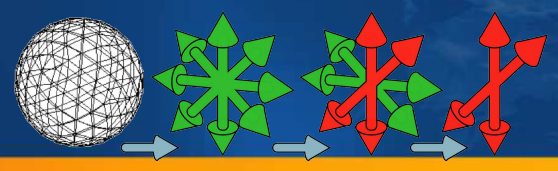




\title{
Fiber Orientation Distribution from Non-Negative Sparse Recovery
}

\author{
Thinhinane Megherbi ${ }^{\dagger}$, Aurobrata Ghosh ${ }^{\star}$, F. Oulebsir Boumghar ${ }^{\dagger}$, Rachid Deriche $^{\star}$ \\ ${ }^{\star}$ Project Team Athena, INRIA, Sophia Antipolis - Méditerranée, France \\ ${ }^{\dagger}$ ParIMed Team, LRPE, USTHB, Algiers, Algeria
}

\section{Introduction}

The Fiber Orientation Distribution (FOD) [3] is a high angular resolution diffusion imaging (HARDI) model for robustly estimating crossing white-matter fiber bundles from q-ball acquisitions. However, its angular resolution depends on the spherical harmonic (SH) / tensor basis order, which implies a large number of acquisitions: 45, 66, 91 for typically used orders such as $8,10,12$. Further, it is still necessary to compute the fiber orientations from the FOD. In the literature two ways have been adopted for this purpose: maxima detection and tensor decomposition.

To overcome this two step approach (FOD estimation + fiber detection), we have proposed a novel FOD model and estimation method based on non-negative sparse recovery [1, 2]. The method has the following advantages: (i) it naturally estimates non-negative FODs, (ii) it computes both the FOD (tensor) and the fiber-orientations together - making tensor decomposition (which is NP-hard) or maxima detection unnecessary, (iii) it doesn't require the number of fiber-compartments to be predefined and (iv) it can estimate very high order FOD tensors from a minimal number of acquisitions (20 or 30). We adopt this method for single shell data of this challenge.

\section{Methods}

In $[1,2]$, we model a single fiber orientation (or an oriented Dirac delta) function as a rank-1 tensor of order $2 n$ : $\mathcal{C}=\lambda \mathbf{c}^{\otimes 2 n}$ and the FOD tensor as the sum of (unknown) $r$ rank-1 tensors: $\mathcal{F}=\sum_{i=1}^{r} \lambda_{i} \mathbf{c}_{i}^{\otimes 2 n}$. Thus, a delta function along $\mathbf{c}$ is described by $C_{n}(\mathbf{u})=\mu_{j 1 j 2 \cdots j 2 n} \mathcal{C}_{j 1 j 2 \cdots j 2 n} u_{j 1} u_{j 2} \cdots u_{j 2 n}=\mathcal{C} \cdot{ }^{(2 n)} \mathbf{u}$, where $\mathbf{u} \in S^{2}$ and $\mu$ is the multiplicity. The FOD is described by $F(\mathbf{u})=\mathcal{F} \cdot(2 n) \mathbf{u}$. This model allows us to choose arbitrarily high tensor orders without increasing the number of unknowns. In this model, the unknown $r$ (the tensor rank), represents the number of fiber compartments and $\lambda_{i}$ represent the weigths of the fiber compartments and cannot be negative. The response function is considered to be a bipolar Watson function $R(\mathbf{q}, \mathbf{u}, b)=e^{-b d_{\|}(\mathbf{q} \cdot \mathbf{u})^{2}}$ and is estimated in voxels with high fractional anisotropy $(\mathrm{FA}>0.8)$ to ensure "single-fiber" configurations.

The FOD estimation is performed by first discretizing the convolution integral $S\left(\mathbf{q}_{j}\right)=\int_{S^{2}} R\left(\mathbf{q}_{j}, \mathbf{u}, b_{j}\right) F(\mathbf{u}) d \mathbf{u}$ and computing the least squares fitting problem (Eq. 1): $\min _{\{\mathbf{w}\}}\|\mathbf{B w}-\mathbf{s}\|^{2}$ st. $\mathbf{w} \geq 0$, where $\mathbf{s}_{j}=S\left(\mathbf{q}_{j}\right)$, $\mathbf{w}_{i}=\lambda_{i}, \mathbf{B}_{i j}=\sum_{l=1}^{L} R\left(\mathbf{q}_{j}, \mathbf{u}_{l}\right)\left(\mathbf{c}_{i} \cdot{ }^{(2 n)} \mathbf{u}_{l}\right) \Delta \mathbf{u}, L$ represents the discretization of the convolution and $d \mathbf{u}$ becomes $\Delta$ u. As $\mathbf{w}_{i}=\lambda_{i}$ cannot cannot be negative and $r$, the length of $\mathbf{w}$, is unknown apriori, we set $\tilde{r}=321,\left\{\mathbf{c}_{i}\right\}=\left\{\tilde{\mathbf{c}}_{i}\right\}$ : a 321-uniform discretization of the unit sphere (which implies an FOD with a 321 crossing-fiber configuration) and solve Eq. 1 with both sparsity and positivity constraints. This is accomplished using the non-negative least squares (NNLS) [2]. Finally, we heuristically clean small fiber weights and unnaturally narrow crossings that can occur due to signal noise and re-fit the resulting configuration to estimate the final FOD (tensor \& fiber-orientations both).

\section{Data \& Results}

Here, we consider the q-ball acquisition with 20 gradient directions and $b=2000 \mathrm{~s} / \mathrm{mm}^{2}$. We estimate $24^{\text {th }}$ order FODs and provide snapshots of both the FOD and the fiber-orientations since both are estimated together.

\section{References}

[1] A. Ghosh, T. Megherbi, F.O Boumghar, and R. Deriche. Fiber orientation distribution from non-negative sparse recovery. In International Symposium on Biomedical Imaging: From Nano to Macro, San Francisco, CA, April 2013.

[2] A. Ghosh and R. Deriche. Greedy NNLS: Fiber Orientation Distribution from Non-Negatively Constrained Sparse Recovery. In Proc. Intl. Soc. Mag. Reson. Med. 22, page 2610, Milan, Italy, May 2014.

[3] J.-D. Tournier et al. Robust determination of the fibre orientation distribution in diffusion MRI: Non-negativity constrained super-resolved spherical deconvolution. NI, 35(4):1459-1472, 2007. 